\title{
An Access Control Model in Lager-Scale P2P File Sharing Systems*
}

\author{
Yue Guang-Xue ${ }^{1,2,3}$, Yu Fei $^{3,4}$, Chen Li-ping $^{1}$, and Chen Yi-jun ${ }^{1}$ \\ ${ }^{1}$ College of Information Engineering, Jiaxing University, 314000 China \\ guangxueyuedyahoo.com.cn \\ ${ }^{2}$ Department of Computer Science and Technology, Huaihua University, 418000 China \\ guangxueyue@126.com \\ ${ }^{3}$ State Key Lab. for Novel Software Technology, Nanjing University, 210093 China \\ yufei@hunau.edu.cn \\ ${ }^{4}$ Jiangsu Provincial Key Laboratory of Computer Information Processing Technology, \\ Suzhou University ,2150063, China \\ hunanyufei@126.com
}

\begin{abstract}
In lager-scale P2P file sharing systems, peers often must interact with unknown or unfamiliar peers without the benefit of trusted third parties or authorities to mediate the interactions. The decentralized and anonymous characteristics of $\mathrm{P} 2 \mathrm{P}$ environments make the task of controlling access to sharing information more difficult. In this paper, we identify access control requirements and propose a trust based access control framework for P2P file-sharing systems. The model integrates aspects of trust and recommendation, fairness based participation schemes and access control schemes.
\end{abstract}

\section{Introduction}

In Peer-to-Peer (P2P) networks, all peers are both consumers and providers of resources and can access each other directly without intermediary peers. Compared with a centralized system, a P2P system provides an easy way to aggregate large amounts of resources residing on the edge of Internet, and it with a low cost of system maintenance[1]. By reason of peers are heterogeneous, some peers might be benevolent in providing services, and some might be even malicious by providing bad services or harming the consumers. There is no centralized node to serve as an authority to monitor and punish the peers that behave badly, malicious peers have an incentive to provide poor quality services for their benefit because they can get away[2]. Some traditional security techniques, such as service providers requiring access authorization, cannot prevent from peers providing variable-quality service. So a major challenge for large-scale P2P file sharing systems is how to establish trust between different peers without the benefit of trusted third parties or authorities. Mechanisms

\footnotetext{
* The research work was supported by the Natural Science Foundation of hunan provincial (No: 05FJ3018) and Open Science Foundation of State Key Lab. for Novel Software Technology, Nanjing University (No: A2006-06).
} 
for trust and reputation can be used to help peers distinguish good or bad partners, which are about generation, discovery, and aggregation of rating information in P2P systems.

\section{Access Control Model Architecture}

There are requirements that an access control model for P2P file sharing systems[3]: Peers in a P2P system are typically loosely coupled, and interacting partners are mostly unknown. Access control mechanisms must provide a mechanism for a host peer to classify users and assign each user different access rights; The incentive for users to join a P2P file-sharing network is the availability and richness of files the system provides. Access control mechanisms needs to give peers the ability to control access to their files it must still encourage them to share their files; The open and unknown characteristics of P2P make it an ideal environment for malicious users to spread unsolicited and harmful content. Access control mechanisms should support mechanisms to limit such malicious spreading, harmful digital content and punish those who are responsible for it.

Peers in P2P file-sharing systems need the autonomy of controlling accesses to their files. Host peer as a stand-alone system where shared files are objects that need to be protected and client peers are subjects who are considered to possess, or gain access rights. Files on a host peer are rated depending on their size and content. They computed from combinations of scores: trust and contribution. The client peer is responsible to collect recommendations that contain the information needed to evaluate its access values for a particular host. After each download transaction, direct trust and direct contribution of both the client peer and host peers are updated accordingly to the satisfaction level of the transaction, which then affect the future evaluation of the access values between these two peers. The architecture sees Figure 1.

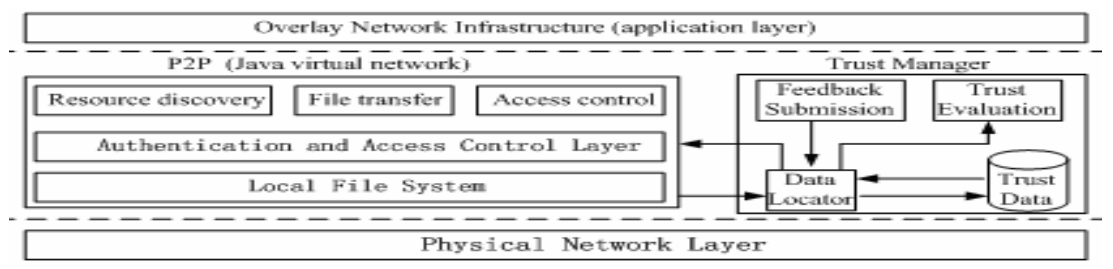

Fig. 1. Overlay architecture of a peer with access control implementation

\subsection{Trust and Reputation Metrics}

Trust is defined as a peer's belief in attributes such as reliability, honesty of the trusted peer. Trust can be broadly categorized by the relationships between the two involved peers. A peer's belief in another peer's capabilities, honesty and reliability based on its own direct experiences, it measures whether a service provider can provide trustworthy 
services; References refer to the peers that make recommendations or share heir trust values. It measures whether a host can provide reliable recommendations.

Reputation of a peer defines an expectation about its behavior, which is based on recommendations received from other peers or information about the peer's past behavior within a specific context at a given time. It can be decentralized, computed independently by each peer after asking other peers for recommendations.

The peer's trust and reputation development cycle see Figure 2[4].

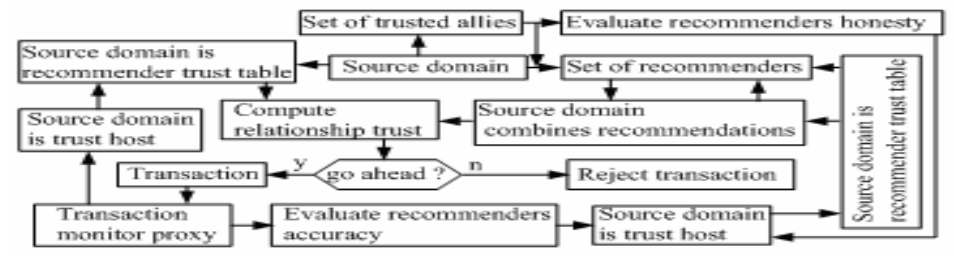

Fig. 2. Trust and reputation development cycle

\subsection{Scoring Scheme for Access Control Model}

As a host peer needs to classify its client peers in order to provide them different access privileges, then model uses a scoring system to differentiate peers based on their behavior in the $\mathrm{P} 2 \mathrm{P}$ network. Hence, after completing the authentication process with the host, a client peer is required to supply its rating certificates for the host to calculate the client's relative access values. They indicate how the host peer perceives the client's trustworthiness and contribution level, which is to ensure the peer is trusted to interact with and to promote fairness in P2P network. There are two sources of information to compute these two values. One source is the host's direct experiences with the client, and other peer's recommendations based on their interactions with the client. Therefore, the access vales are evaluated via combinations of four types of scores: direct trust, indirect trust, direct contribution and indirect contribution. It tries to reduce the problem of unfair trading. Figure 3 represents procedures in a typical interaction between a host and a client in our framework. It can be seen the scheme adopts more of a "push" approach.

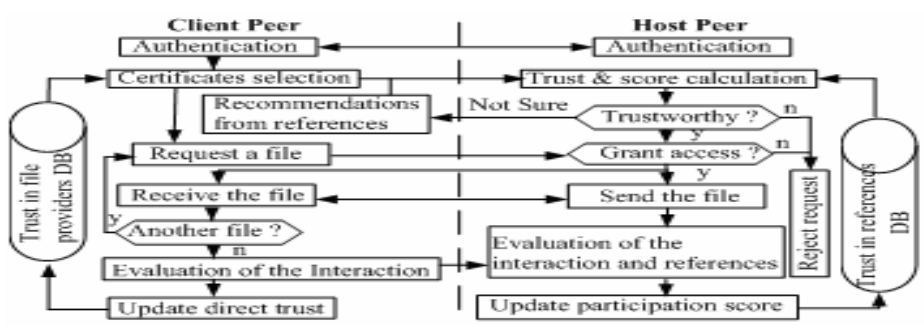

Fig. 3. Flow chart of an interaction between a host peer and a client peer 
The Algorithm of peers constructing a trust graph as follows

Step1 suppose peer $P_{t}$ is evaluating the trustworthiness of peer $P_{k}$ and $P$ is the set of peers being visited. $\left\{P_{i}, P_{j}\right\}$ denotes a referral $r$ to peer $P_{j}$ returned from peer $P_{i}$. $P$ is a finite set of peers $\left\{P_{l}, \ldots, P_{n}\right\}$, and $R$ is a set of referrals $\left\{r_{l}, \ldots, r_{n}\right\}$.

Step2 for $\left(\forall P_{i} \notin P\right.$ and $P_{i}$ has not been queried) do if (Trust $\left(P_{i}\right)<$ TrustBound) then $\Rightarrow P_{t}$ queries $P_{i}$

Step3 if $\left(P_{i}\right.$ is a witness of $\left.P_{k}\right)$ then $\Rightarrow P_{i}$ returns the rating about $P_{k}$ to $P_{t}$, interation, compute trust; else

Step4 for $\left(\forall r=\left\{P_{i}, P_{j}\right\}\right.$ from $\left.P_{i}\right)$ do, computer recommendation

Step5 if $\left(P_{j} \notin P\right)$ then $\Rightarrow r \rightarrow R, P_{j} \rightarrow P$; else

Step6 ignore referral $r$

Step7 evaluation of the interaction, references and update $D B s$.

Therefore, it could effectively solve the follows problems

(1) A client submits other peers' recommendations to a host in the form of rating certificates.

(2) It is reasonable to expect that the greater the number of peers that the client has interacted with, the more recommendations it has and the higher its indirect trust as rated by the host peer.

(3) Avoid specify a required number of recommending peers. In general, this can vary from peer to peer and depending on the type of transaction.

\section{Conclusion}

The proposed access control model and score systems help to classify both known and unknown visitors according to their trustworthiness and contribution. The implemented contribution scores work effectively as a payment scheme; giving incentive for users to share their resources and safeguarding the fairness of service exchange in a P2P system. The proposed mechanisms for evaluating a transaction not only help to differentiate poorly performing peers from good ones but also ensure that malicious peers are punished and isolated.

\section{References}

1. Parameswaran M, Susarla A, Whinston AB. P2P networking: An information-sharing alternative. Computing Practices, 2001,34(7):31-38.

2. Yao Wang, Julita Vassileva. Bayesian Network-Based Trust Model. Proceedings of the IEEE/WIC International Conference on Web Intelligence (WI'03)

3. Huu Tran, Michael Hitchens, Vijay Varadharajan, Paul Watters. A Trust based Access Control Framework for P2P File-Sharing Systems. Proceedings of the 38th Hawaii International Conference on System Sciences -2005.

4. Farag Azzedin, Muthucumaru Maheswaran. Trust Modeling for Peer-to-Peer based Computing Systems. Proceedings of the International Parallel and Distributed Processing Symposium (IPDPS'03). 